\title{
BOUNDS ON THE EIGENVALUES OF THE LAPLACE AND SCHROEDINGER OPERATORS
}

\author{
BY ELLIOTT LIEB 1
}

Communicated by I. M. Singer, April 19, 1976

If $\Omega$ is an open set in $\mathbf{R}^{n}$, and if $\widetilde{N}(\Omega, \lambda)$ is the number of eigenvalues of $-\Delta$ (with Dirichlet boundary conditions on $\partial \Omega$ ) which are $\leqslant \lambda(\lambda \geqslant 0)$, one has the asymptotic formula of Weyl [1], [2]: $\lim _{\lambda \rightarrow \infty} \lambda^{-n / 2} \widetilde{N}(\Omega, \lambda)=C_{n}|\Omega|$. Here $|\Omega|$ is the volume of $\Omega$ and $C_{n}=(4 \pi)^{-n / 2} \Gamma(1+n / 2)^{-1}$. The same holds [3] if $\mathbf{R}^{n}$ is replaced by a Riemannian manifold, $M$, with $|\Omega|$ being the Riemannian volume and $\Delta$ being the Laplace-Beltrami operator. One purpose of this note is to state that there often exist bounds of the form

$$
\begin{aligned}
& \widetilde{N}(\Omega, \lambda) \leqslant D_{n} \lambda^{n / 2}|\Omega|, \forall \lambda \geqslant 0, \forall \Omega \subset M, \\
& \widetilde{N}(\Omega, \lambda) \leqslant\left(D_{n} \lambda^{n / 2}+E_{n}\right)|\Omega|, \quad \forall \lambda \geqslant 0, \forall \Omega \subset M,
\end{aligned}
$$

with $D_{n}, E_{n}$ independent of $\lambda$ and $\Omega$ and depending only on $M$. (1a) holds for noncompact $M$ if condition (8), below, holds. In particular, (1a) holds for $\mathbf{R}^{n}$ and for homogeneous spaces with curvature $\leqslant 0$. (1b) always holds for compact $M$, and it also holds for noncompact $M$ if condition (9) holds.

REMARK. There is an asymptotic formula [4], [5]: $\widetilde{N}(\Omega, \lambda)=$ $C_{n} \lambda^{n / 2}|\Omega|+O\left(\lambda^{(n-1) / 2}\right)$. While this has the correct limiting constant, $C_{n}$, the remainder, $O(\cdot)$, can get very large if $\Omega$ is very irregular. The remainder is not bounded by a universal constant times $|\Omega| \lambda^{(n-1) / 2}$ or even $|\Omega| \lambda^{n / 2}$. Our emphasis is different. By introducing $D_{n} \geqslant C_{n}$ we have a bound which is universal in the sense that it depends on $M$ but not on $\Omega \subset M$; in particular, (1) is applicable to unbounded $\Omega$.

A second, closely related problem is to estimate $N_{\alpha}(V)=$ number of nonpositive eigenvalues of the Schroedinger operator $-\Delta+V(x)$ on $L^{2}(M)$ which are $\leqslant \alpha \leqslant 0$. There exists an asymptotic formula [6], [7] , [8] for suitably regular $V$ :

$$
\lim _{\gamma \rightarrow \infty} \gamma^{-n / 2} N_{\gamma \alpha}(\gamma V)=C_{n} \int_{M}[V(x)-\alpha]_{-}^{n / 2} d x
$$

AMS (MOS) subject classifications (1970). Primary 58G99, 35J05, 35J10, 35P15, 35P20; Secondary 47F05, $81 \mathrm{A09}, 81 \mathrm{~A} 45$.

${ }^{1}$ Work supported by U. S. National Science Foundation grant MCS 75-21684. 
where $V_{-}=1 / 2(|V|-V)$, and $d x$ is the Riemannian volume element. Our new, nonasymptotic result is

$$
N_{\alpha}(V) \leqslant L_{n} \int_{M}[V(x)-\alpha]_{-}^{n / 2} d x, \quad \forall \alpha, V
$$

when $M$ satisfies (8) and $\operatorname{dim}(M) \geqslant 3$. [(3) was obtained simultaneously and independently by M. Cwikel [9]; his estimate for $L_{n}$ is not as sharp as ours, however. When $n=3$, our $L_{3}=.116$ and it is known that $L_{3} \geqslant .078$.]

The connection between $\widetilde{N}(\Omega, \lambda)$ and $N_{\alpha}(V)$ is the following elementary consequence of the min-max principle:

$$
\tilde{N}(\Omega, \lambda) \leqslant N_{\alpha}\left((\alpha-\lambda) \chi_{\Omega}\right), \quad \forall \alpha \leqslant 0
$$

where $\chi_{\Omega}$ is the characteristic function of $\Omega$. Thus (3) implies $\tilde{N}(\Omega, \lambda) \leqslant$ $L_{n} \lambda^{n / 2}|\Omega|$ for $\operatorname{dim}(M) \geqslant 3$. Another important consequence of the min-max principle is

$$
N_{\alpha}(V) \leqslant N_{\alpha+\beta}\left(-(V+\beta)_{-}\right), \quad 0 \leqslant \beta \leqslant-\alpha .
$$

Consequently, one need consider only the case $V=-V_{-}$in (3).

The asymptotic formula (2) has been extended to $V_{-} \in L^{n / 2+\epsilon} \cap L^{n / 2-\epsilon}$ by Simon [10]. Using his methods and (3), one easily extends (2) to all $V_{-} \in$ $L^{n / 2}$.

Results (1) and (3) are corollaries of the following

Theorem. Let $f:[0, \infty) \rightarrow[0, \infty)$ be convex and polynomially bounded at infinity and satisfy

$$
\int_{0}^{\infty} t^{-1} e^{-t} f(t) d t=1
$$

For $t>0$, let $G(x, y ; t)$ be the kernel of $e^{t \Delta}$, i.e. the fundamental solution of the heat equation on the Riemannian manifold $M$. Then, for $\alpha \leqslant 0$,

$$
N_{\alpha}(V) \leqslant \int_{M} d x \int_{0}^{\infty} t^{-1} e^{\alpha t} G(x, x ; t) f\left(t V_{-}(x)\right) d t .
$$

Our proof of this theorem uses the Wiener integral in an essential way and will be published elsewhere.

To apply (7) we choose $f(t)=0, t \leqslant a, f(t)=b(t-a), t \geqslant a$, for some $a, b>0$ such that (6) holds. To prove (3), we assume

$$
G(x, x ; t) \leqslant A t^{-n / 2}, \quad \forall x \in M, \forall t>0 .
$$

This holds for $\mathbf{R}^{n}\left(A=(4 \pi)^{-n / 2}\right)$ and for homogeneous spaces with curvature $\leqslant 0$. Next we use (5) with $\beta=-\alpha$ and then (7) with $\alpha=0$.

To prove (1a) we assume (8). For (1b) we require a bound of the form

$$
G(x, x ; t) \leqslant A t^{-n / 2}+B, \quad \forall x \in M, \forall t>0,
$$

which always holds for compact $M$, for example. In either case, using (4) and (7) with $\alpha=-\lambda$, 


$$
\tilde{N}(\Omega, \lambda) \leqslant \int_{\Omega} d x \int_{0}^{\infty} t^{-1} e^{-t / 2} G\left(x, x ;(2 \lambda)^{-1} t\right) f(t) d t .
$$

The author is most grateful to B. Simon for many helpful suggestions during the course of this work. He is also indebted to I. M. Singer for remarks on Riemannian manifolds.

ADDED IN PROOF. I have recently become aware of the paper of G. V. Rozenbljum, Dokl. Akad. Sci. SSSR 202 no. 5 (1972), 1012-1015 (MR 45 \#4216) in which a proof of (3) is announced. Rozenbljum's method is completely different, however, and his estimate for $L_{n}$ does not appear to be as sharp.

\section{REFERENCES}

1. H. Weyl, Das asymptotische Verteilungsgesetz der Eigenwerte linearer partieller Differentialgleichungen, Math. Ann. 71 (1911), 441-469.

2. M. Kac, Can one hear the shape of a drum? Slaught Memorial Papers, no. 11, Amer. Math. Monthly 73 (1966), no, 4, part II, 1-23. MR 34 \#1121.

3. S. Minakshisundaram and A. Pleijel, Some properties of the eigenfunctions of the Laplace-operator on Riemannian manifolds, Canad. J. Math 1 (1949), 242-256. MR 11, 108.

4. V. G. Avakumović, Über die Eigenfunktionen auf geschlossenen Riemannschen Mannigfaltigkeiten, Math. Z. 65 (1956), 327-344. MR 18, 316.

5. L Hörmander, The spectral function of an elliptic operator, Acta Math. 121 (1968), 193-218.

6. A. Martin, Bound states in the strong coupling limit, Helv. Phys. Acta 45 (1972), 140-148.

7. H. Tamura, The asymptotic eigenvalue distribution for non-smooth elliptic operators, Proc. Japan Acad. 50 (1974), 19-22. MR 51 \#1153.

8. M. S. Birman and V. V. Borzov, On the asymptotic formula for the discrete spectrum of certain singular differential operators, Problemy Mat. Fiz., vyp. 5, Izdat. Leningrad. Gos Univ. Leningrad, 1971 , pp. 24-38 = Topics in Math. Phys., no. 5, Plenum Press, New York, 1972, pp. 19-30; Consultants Bureau Transl., pp. 1-18. MR 46 \#726.

9. M. Cwikel, Weak type estimates for singular values and the number of bound states of Schroedinger operators, Institute for Advanced Study, Princeton, N. J., 1976 (preprint).

10. B. Simon, Analysis with weak trace ideals and the number of bound states of Schrödinger operators, Trans. Amer. Math. Soc. (to appear).

DEPARTMENT OF MATHEMATICS AND PHYSICS, PRINCETON UNIVERSITY, PRINCETON, NEW JERSEY 08540 